\title{
A Review of the Burden of Trauma Pain in Emergency Settings in Europe
}

Patrick D. Dißmann - Maxime Maignan - Paul D. Cloves •

Blanca Gutierrez Parres - Sara Dickerson - Alice Eberhardt

Received: October 5, 2017 / Published online: June 2, 2018

(C) The Author(s) 2018

\section{ABSTRACT}

Trauma pain represents a large proportion of admissions to emergency departments across Europe. There is currently an unmet need in the treatment of trauma pain extending throughout the patient journey in emergency settings. This

Enhanced digital features To view enhanced digital features for this article go to https://doi.org/10.6084/ m9.figshare.6340571.

Electronic supplementary material The online version of this article (https://doi.org/10.1007/s40122018-0101-1) contains supplementary material, which is available to authorized users.

P. D. Dißmann

Emergency Department, Klinikum Lippe GmbH, Detmold, Germany

M. Maignan

Emergency Department, Grenoble Alpes University

Hospital, CHUGA, Grenoble, France

P. D. Cloves

South East Coast Ambulance Service, Brighton, UK

B. Gutierrez Parres

Emergency Department, Puerta de Hierro University

Hospital, Madrid, Spain

S. Dickerson $(\bowtie)$

Mundipharma International Limited, Cambridge, UK

e-mail: sara.dickerson@mundipharma.com

A. Eberhardt

Mundipharma GmbH, Limburg, Germany review aims to explore these unmet needs and describe barriers to the delivery of effective analgesia for trauma pain in emergency settings. A comprehensive, qualitative review of the literature was conducted using a structured search strategy (Medline, Embase and Evidence Based Medicine Reviews) along with additional Internet-based sources to identify relevant human studies published in the prior 11 years (January 2006-December 2017). From a total of 4325 publications identified, 31 were selected for inclusion based on defined criteria. Numerous barriers to the effective treatment of trauma pain in emergency settings were identified, which may be broadly defined as arising from a lack of effective pain management pan-European and national guidelines, delayed or absent pain assessment, an aversion to opioid analgesia and a delay in the administration of analgesia. Several commonly used analgesics also present limitations in the treatment of trauma pain due to the routes of administration, adverse side effect profiles, pharmacokinetic properties and suitability for use in pre-hospital settings. These combined barriers lead to the inadequate and ineffective treatment of trauma pain for patients. An unmet need therefore exists for novel forms of analgesia, wider spread use of available analgesic agents which overcome some limitations associated with several treatment options, and the development of protocols for pain management which include patient assessment of pain. 
Funding: Mundipharma International Ltd.

Keywords: Ambulance; Analgesia; Emergency; Pain; Trauma

\section{INTRODUCTION}

Management of trauma pain by healthcare professionals (HCPs) in the emergency department (ED) and prehospital settings is a crucial element of care. Approximately 38 million people across Europe visit the hospital ED each year due to injuries, with 5.3 million of these patients admitted for further treatment [1]. Pain is often the main complaint of trauma patients and is reported by up to $70 \%$ of patients in prehospital settings and 91\% in EDs [2-4].

However, inadequate relief of trauma pain is commonly reported by patients in the EU and beyond [5]. For example, in a large, multicenter study conducted in the US and Canada, $74 \%$ of patients were discharged from the ED in moderate or severe pain [6]. Moderate-to-severe pain is also commonly reported by patients discharged from European EDs [7]. In Europe, the treatment for trauma pain is largely similar between the pre-hospital setting and the ED, mainly consisting of paracetamol, non-steroidal anti-inflammatory drugs (NSAIDs), nitrous oxide $\left(\mathrm{N}_{2} \mathrm{O}\right)$, and opioids [3, 8-10]. Current use of these analgesics may be considered inadequate. Indeed, prospective data from Norwegian and Italian EDs indicated only 14 and 32\% of patients with moderate-to-severe pain received analgesia, respectively [10, 11]. Suboptimal assessment and management of trauma pain has also been reported by emergency medical services in prehospital settings [3, 12]. The impact associated with lack of effective pain control also extends beyond the patient's perspective to the wider emergency setting as HCPs are, in turn required to manage increased levels of pain which impacts resources [4]. Consequently, there appears to be an unmet need for a safe, timely, and efficacious treatment for trauma pain in emergency settings.
We conducted a qualitative review of published literature with the aim of identifying current barriers to the effective management of trauma pain in Europe. Based on these findings, we sought to identify potential areas for improvement in the management of trauma pain in emergency settings.

\section{METHODS}

A literature search was conducted to identify publications reporting current treatment approaches for trauma pain in emergency settings in Europe (including both pre-hospital and EDs), the limitations of these therapies and other barriers to effective pain control. An integrative review framework was used. This approach enables evaluation of heterogeneous studies, thereby providing comprehensive methodology to assess a particular healthcare phenomenon [13]. The following computerized bibliographic databases were searched using the OVID search engine: Medline, Embase, and the Evidence-Based Medicine Reviews. The search was limited to human studies published in English language in the past 11 years (January 01, 2006-December 31, 2017). Combinations of terms were utilized such as (analgesia or acute pain or injury or trauma pain) and (emergency services or emergency department or pre-hospital) OR (treatment pathway or standard of care) OR (cost or economic or financial) OR (quality of life or treatment satisfaction or social cost). Retrieved abstracts were assessed for relevance against a pre-defined inclusion and exclusions criteria, agreed by the co-authors prior in order to establish the objectives of this literature analysis (Table 1). The search strategy was agreed by all co-authors, and all co-authors were involved in the final selection and appraisal of the papers. All types of studies were captured in this qualitative review, including randomized controlled trials (RCTs), observational studies, review articles, and treatment guidelines.

An additional search of Internet-based sources (websites of the World Health Organization, NHS Choices, and College of 
Table 1 Inclusion and exclusion criteria used in the literature review

\begin{tabular}{|c|c|}
\hline Inclusion criteria & Exclusion criteria \\
\hline Publications were included which: & Publications were excluded which: \\
\hline $\begin{array}{l}\text { 1. Discussed the prevalence or incidence of pain in } \\
\text { emergency settings }\end{array}$ & $\begin{array}{l}\text { 1. Did not report on pain or treatments for pain } \\
\text { 2. Focused on long-term chronic pain }\end{array}$ \\
\hline $\begin{array}{l}\text { 2. Discussed pain in relation to time and duration of pain } \\
\text { before relief in emergency settings }\end{array}$ & $\begin{array}{l}\text { 3. Related solely to the treatment of trauma pain in specific } \\
\text { patient groups, including: pediatrics, elderly, pregnant, or }\end{array}$ \\
\hline $\begin{array}{l}\text { 3. Reported treatment pathways for mild, moderate, and } \\
\text { severe trauma pain in emergencies. This could be recorded } \\
\text { also as "pain induced by trauma", "trauma-induced pain", or } \\
\text { "acute pain from fractures" }\end{array}$ & $\begin{array}{l}\text { patients with reduced consciousness (papers that included } \\
\text { subgroups of populations [e.g., elderly or pediatric } \\
\text { patients] within a range of individuals were not excluded) } \\
\text { 4. Focused on the treatment of pain from major trauma }\end{array}$ \\
\hline $\begin{array}{l}\text { 4. Captured treatment patterns and pain management of } \\
\text { patients with moderate-to-severe trauma pain }\end{array}$ & 5. Did not have a European focus \\
\hline \multicolumn{2}{|l|}{$\begin{array}{l}\text { 5. Described the limitations of current treatments for } \\
\text { trauma pain in emergency settings }\end{array}$} \\
\hline $\begin{array}{l}\text { 6. Discussed the burden of trauma pain in an emergency } \\
\text { setting on healthcare providers }\end{array}$ & \\
\hline
\end{tabular}

Emergency Medicine) was conducted to identify relevant gray literature, i.e., research produced by organizations outside of traditional publishing channels. This search was conducted using no pre-defined search criteria and included both publicly available information and peer-reviewed publications that may not yet be indexed in databases such as PubMed or Embase because of their recent publication date or because they were published in journals that are not indexed within these databases. Additional references were identified by targeted searching for information to corroborate expert knowledge shared by authors of working practices in their respective countries. Investigative questions regarding the standard of care in the treatment of trauma pain and barriers to effective management of trauma pain were developed and addressed using evidence collated from the identified studies. This article is based on previously conducted studies and does not contain any studies with human participants or animals performed by any of the authors.

\section{RESULTS}

\section{Search Results}

Our searches revealed a sparsity of relevant European literature on trauma pain management. From 4325 publications identified in the literature search, 31 were selected for inclusion in this analysis based on the inclusion and exclusion criteria described in Table 1 (Fig. 1). The publications identified by the primary literature search included eight observational patient assessments, eight literature reviews, eight patient chart reviews, three RCTs (and associated subanalyses), one pain management guideline, and one HCP questionnaire. Thirteen additional publications were identified through a grey literature search of freely available sources, including six literature reviews, three observational patient assessments, one RCT, one pain management guideline and two patient information Web pages. Details of these 44 publications are summarized in the supplementary material (Supplementary Table 1 and Supplementary Table 2). 


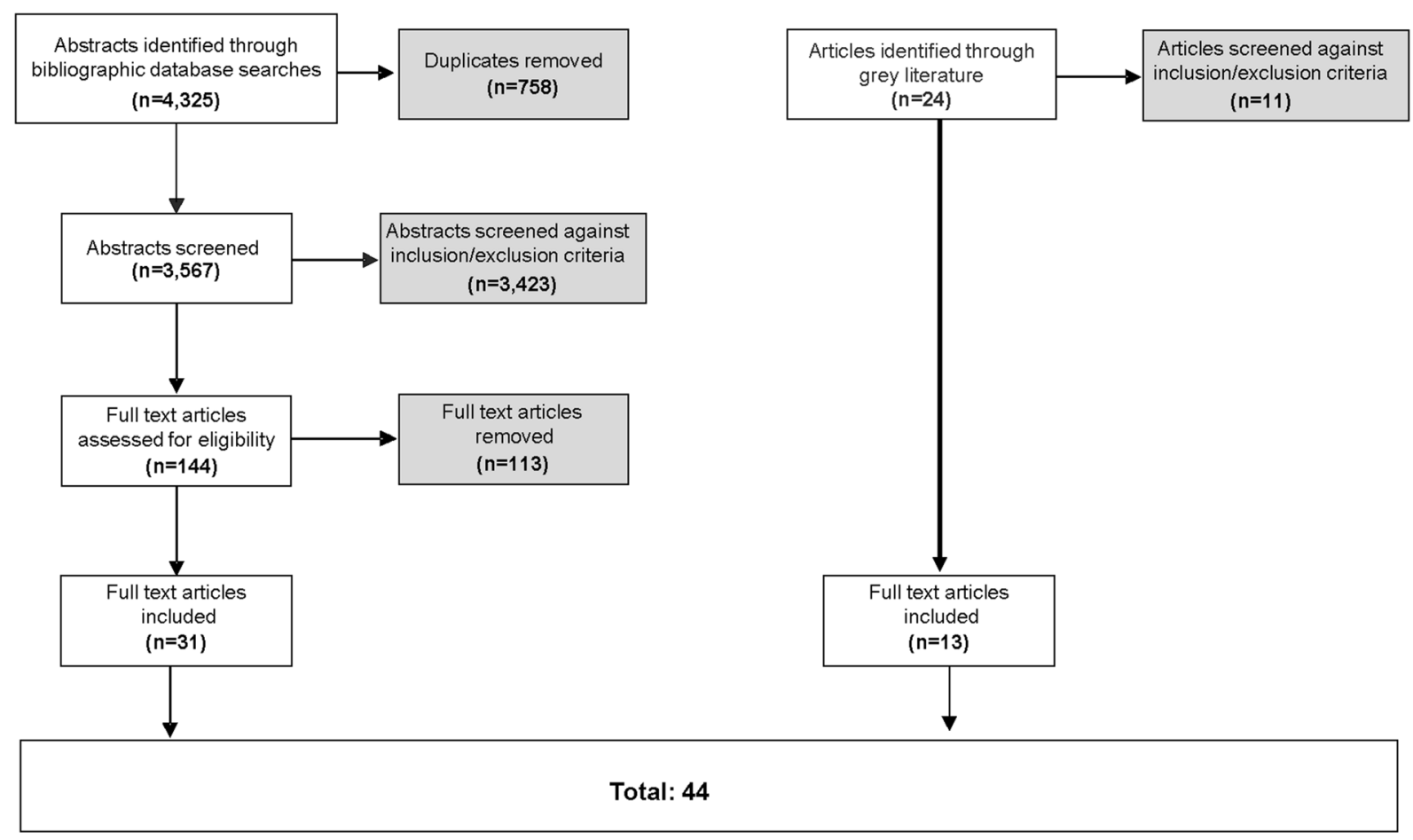

Fig. 1 Schematic of publications included in the literature review for trauma pain

What is the Standard of Care in the Treatment of Trauma Pain?

Common analgesics used in the pre-hospital and ED settings in Europe include paracetamol, NSAIDs, $\mathrm{N}_{2} \mathrm{O}$, and opioids $[3,8,9]$. The type of analgesic used can depend on trauma type, pain severity or triage system in the ED $[8,9,14]$. Regional blocks, for example local anesthesia and peripheral nerve blocks, may also be administered in the treatment of trauma pain $[8,15,16]$. These treatments may reduce the need for rescue/additional analgesic treatment [17]. Although not a common theme in the literature identified in this search, non-pharmacological approaches also play an important role in ameliorating trauma pain, for example immobilizing limbs and applying dressings or ice packs, and may be used in conjunction with drug therapy $[3,8]$. Some treatment options have limitations which may hinder effective pain relief in emergency settings, and are discussed below.

\section{What are the Pharmacological Treatment Options for Mild to Moderate Pain?}

Paracetamol and/or NSAIDs are often used in first-line treatment of mild to moderate pain with the route of administration, usually orally or intravenous (IV), depending on the setting and patient needs $[8,18-20]$. Commonly prescribed NSAIDs in Europe include ibuprofen, diclofenac, and naproxen $[8,21]$. In a recent double-blind study, paracetamol was found to be non-inferior to diclofenac as an analgesic for acute, minor musculoskeletal trauma [22]. However, paracetamol does not have the antiinflammatory properties of NSAIDs.

$\mathrm{N}_{2} \mathrm{O}$ is an inhaled, rapid-onset, short-acting analgesic commonly used in emergency settings $[8,23] . \mathrm{N}_{2} \mathrm{O}$ has been used as an analgesic in pre-hospital settings and EDs for many years, where its short duration of action $(\leq 5 \mathrm{~min}$ of analgesia) is well suited for the treatment of acute trauma pain [24].

Metamizole (dipyrone) is a non-opioid analgesic and its use in emergency settings varies considerably across Europe. Metamizole is banned in some countries (e.g., the UK, Sweden, 
and some countries outside of Europe including the US) due to concern over myelotoxicity, but its use is widespread across others (e.g., Spain and Germany, based on authors discussions) [25-27]. A recent systematic review indicated more large-scale studies are required to better understand the risks and benefit of metamizole relative to other analgesics [27].

Weak opioids such as codeine and tramadol are also used to treat moderate trauma pain $[8,28]$. Tramadol acts at $\mu$-opioid receptors and inhibits the reuptake of serotonin and norepinephrine [29]. This provides an atypical analgesic effect to that usually experienced with this class of pain relief products along with less severe side effect profile. Typical opioid side effects are uncommon with tramadol use, making this analgesic a useful analgesic option $[24,29]$.

\section{What are the Pharmacological Treatment Options for Severe Pain}

Opioids provide effective analgesia for severe trauma pain and are available by several routes of administration, including IV, or intranasal (IN), intraosseous (IO), subcutaneous (SC), and per os (PO). While morphine is most commonly used in emergency settings across Europe for severe pain, use of other opioids including fentanyl and oxycodone is also common $[3,28,30,31]$.

Ketamine can also provide effective analgesia for severe trauma pain [12, 28, 31]. Although the exact mechanism of action is largely unknown, its wide therapeutic index, cardiovascular stability, and lack of respiratory depression make ketamine attractive for use in the pre-hospital setting [28]. The dissociative effect associated with ketamine also makes it an effective treatment for trauma pain, although safety concerns over psychological manifestations and long-term psychotomimetic effects have been raised [32].

Low-dose methoxyflurane, a non-opioid, volatile fluorinated hydrocarbon, is administered via the hand-held Penthrox ${ }^{\circledR}$ inhaler. While use of methoxyflurane for general anesthesia was discontinued due to renal safety concerns, administration of sub-anesthetic concentrations for short periods is not associated with nephrotoxicity [33]. Low-dose methoxyflurane has been used extensively in emergency settings in Australia and New Zealand for over 30 years and was recently approved in some European countries (including Belgium, France, Ireland, and the UK) for the emergency relief of moderate-to-severe pain in conscious adults with trauma and associated pain $[34,35]$. In a double-blind trial (STOP!) of adults and adolescents presenting at the ED with moderate pain arising from minor trauma, methoxyflurane provided greater improvements in pain versus placebo at timepoints from 5 to $20 \mathrm{~min}$ and was well tolerated (adverse events were mild and transient) [36]. Subgroup analysis of the STOP! trial in adults and those with contusions and lacerations confirmed these findings [37-39].

Multimodal pain management using two or more drugs with differing modes of action has an important role alleviating trauma pain. For example, paracetamol, NSAIDs, or ketamine may be used in combination with opioids $[8,12,19,29]$. Evidence outside the setting of trauma pain suggests this approach can reduce the dose of opioids required (opioid-sparing effect) [40].

\section{What are the Barriers to Effective Management of Trauma Pain?}

The journey of a patient with trauma pain, including ambulance care, triage, and physician assessments in the ED, presents several stages where barriers to effective management may exist (Fig. 2). The barriers identified in this literature analysis are discussed in detail below, including limitations of currently available therapies, HCP perceptions regarding opioids, lack of national emergency pain treatment guidelines in most European countries, and inadequate pain assessment in emergency settings. These findings highlight that significant cultural changes are needed in emergency medicine to improve trauma pain management and incorporate a more patient-centric approach. 


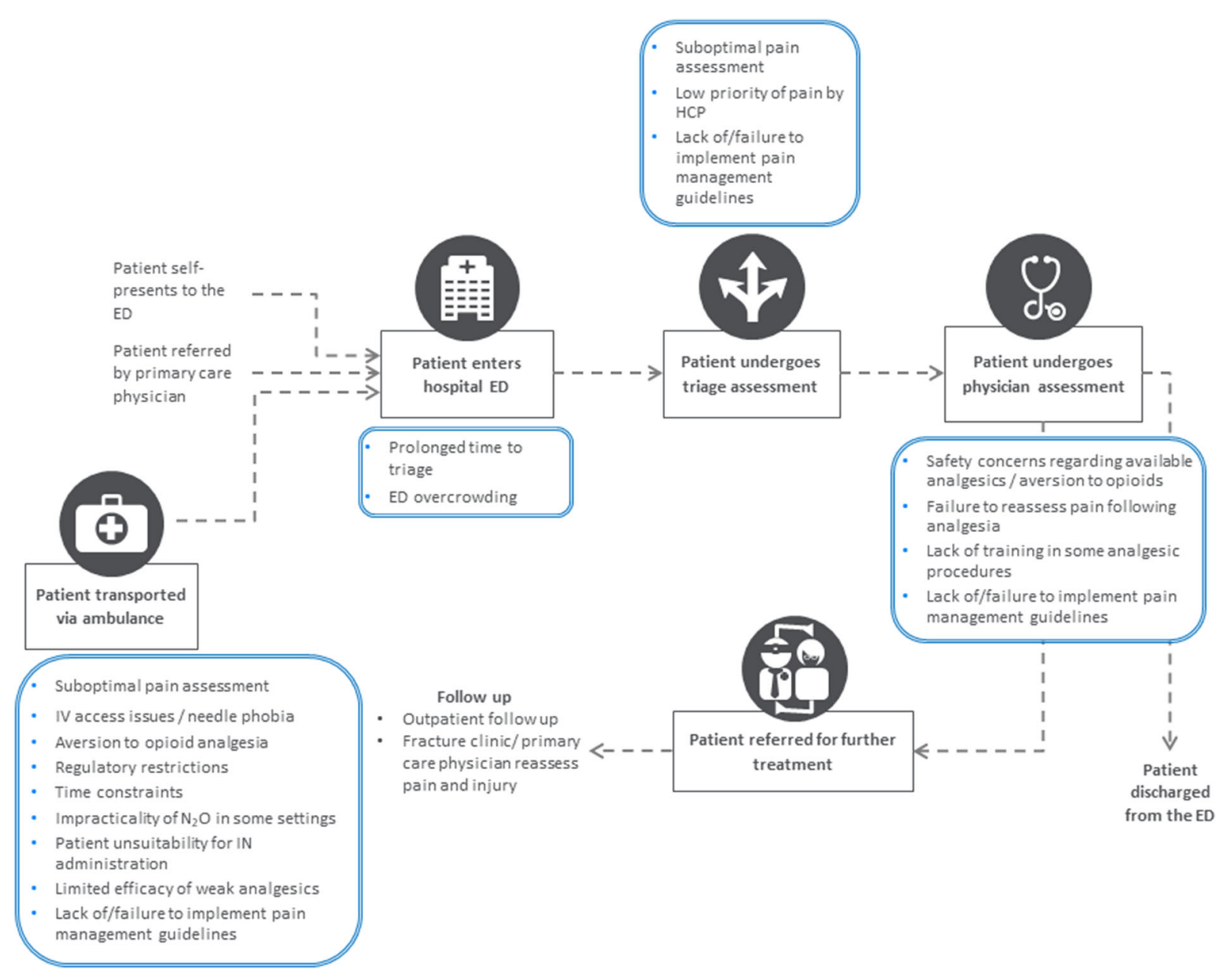

Fig. 2 Treatment pathway and barriers to effective management of trauma pain in Europe. ED emergency department, $H C P$ healthcare professional, $I N$ intranasal,

\section{What are the Limitations of Current Treatment Options for Trauma Pain?}

The type of analgesic recommended for use in the treatment of pain in emergency settings can depend on trauma type, pain severity, and triage system in the ED [8, 14, 19]. Limitations associated with commonly used analgesics were identified, which underscore the need for alternate analgesics to address trauma pain (Fig. 2).

\section{Difficulties Associated with Routes of Administration}

The route of administration of analgesics in the treatment of trauma pain may present several limitations. IV analgesia is often the most
$I V$ intravenous. Barriers to effective management of trauma pain are detailed in boxes

common route of administration in emergency settings and provides fast-onset pain relief [19]. However, IV analgesics can be difficult to administer in some circumstances, such as on the scene of accidents. Problems can also occur in attempting to gain IV access in cold weather in pre-hospital settings, or in patients with difficult vein access, causing further discomfort to distressed individuals and delaying onset of analgesia. Furthermore, in some countries, including Denmark, many paramedics are not authorized to administer IV medication [41]. Studies outside of this Europe-focused literature search have also reported associations between IV access difficulties and increased on-scene time for ambulance crews, and consequent ED 
crowding as attention of HCPs is diverted [42-44].

IV and other methods of administering medications by injection can be painful and may not be suitable for use in patients with 'needle-phobia'. In addition, while SC administration of analgesics can provide rapid and titratable pain relief, it is unsuitable for edematous or hypovolemic patients. IM administration does not allow for dose titration or adjustment, potentially resulting in ineffective and indeterminate levels of analgesia. Furthermore, IO administration of analgesia requires prior placement of IO access, which causes the patient further pain and is not used in common practice. Many trauma patients eligible for local anesthesia or regional nerve blocks fail to receive such treatment, which has been attributed in part to inadequate training of HCPs in these procedures $[15,16]$.

The use of IN analgesia in emergency settings is less invasive compared to IV administration. However, IN analgesia can cause administration issues in patients with facial trauma such as epistaxis, blocked nose, and accidental swallowing [45]. In such individuals, this may result in a suboptimal dose of analgesia and therefore ineffective treatment of trauma pain.

\section{Opioid Safety Profile}

Opioids are considered the benchmark for analgesia of severe pain in emergency settings. However, opioids are associated with a challenging safety profile, including risks of respiratory depression, cardiovascular events, nausea, and vomiting [28,46]. As a result of associated adverse events (particularly respiratory depression), patients require prolonged monitoring and observation following opioid administration, therefore increasing the HCP workload and patient length of stay [14].

\section{Limited Efficacy of Weak Analgesics}

Weaker analgesics such as metamizole, paracetamol, and NSAIDs are limited in their ability to treat moderate-to-severe pain. Pain as a result of trauma can quickly escalate in severity, and therefore the use of weaker analgesics may provide ineffective analgesia.
The widespread availability of paracetamol and some NSAIDs without prescription in Europe means that many patients may have selfmedicated with these drugs prior to presenting at the ED [47]. As overdosing is associated with serious side effects, inquiry should be made regarding recent use of over-the-counter paracetamol-containing preparations before prescribing [8, 47]. Of note, a recent review of observational studies (which was not focused on emergency trauma pain) revealed considerable toxicity with paracetamol at the upper end of standard analgesic doses [48]. Limitations of metamizole analgesia are largely associated with its uncertain safety profile, resulting in a ban in countries such as Sweden and the UK [26, 27]. A US-based review of pain management in ED also discussed how patients may become frustrated if the same analgesia they have already taken is offered again in the ED [24].

\section{Practicalities of $\mathrm{N}_{2} \mathrm{O}$}

$\mathrm{N}_{2} \mathrm{O}$ analgesia may be unsuitable for some patients, for example individuals with pneumothorax or facial/head trauma $[8,23]$. The varied efficacy experienced by patients receiving $\mathrm{N}_{2} \mathrm{O}$ also means there are a limited number of non-responders to this treatment [23].

Despite its proven analgesic effect, operational issues can hinder the use of $\mathrm{N}_{2} \mathrm{O}$ as treatment involves large amounts of equipment (such as cylinders and breathing apparatus) and transport of bulky cylinders of premixed $\mathrm{N}_{2} \mathrm{O}$ and oxygen [34]. The mix of oxygen and $\mathrm{N}_{2} \mathrm{O}$ can separate at cold temperatures and must be stored at temperatures above $10^{\circ} \mathrm{C}$ for at least $24 \mathrm{~h}$ prior to use to avoid potentially hypoxic concentrations being delivered as the cylinder empties [34]. The equipment required for $\mathrm{N}_{2} \mathrm{O}$ treatment can also limit the accessibility of analgesia in some situations (for example to patients in remote locations) and also impacts the volume of equipment available in an ambulance. It has been proposed that methoxyflurane, which is delivered in the handheld Penthrox ${ }^{\circledR}$ inhaler, can overcome some of the limitations associated with $\mathrm{N}_{2} \mathrm{O}$ treatment as portability is particularly desirable for emergency care in remote locations or 
rescue helicopter missions, as well for urban paramedics who carry heavy backpacks $[34,35]$.

\section{Lack of Effective Pain Management Guidelines} Pain management guidelines are important for the effective management of trauma pain as this guidance is intended to ensure all patients receive appropriate pain relief. This literature review identified no pan-European guidelines for the treatment of trauma pain in prehospital or hospital settings, and also a lack of consistent guidelines at a national level. Indeed, only two published guidelines were identified by our literature analysis: institution clinical practice guideline (Switzerland) and College of Emergency Medicine (UK) [8, 19]. These guidelines broadly reflect US evidence-based guidance for prehospital trauma analgesia [49].

In 1986, the World Health Organization (WHO) issued a 'pain relief ladder' outlining analgesics recommended for relief from cancer pain in adults (Fig. 3) [50]. This format may provide a basis for the recommendation of opioids in the treatment of moderate-to-severe trauma pain in both the pre-hospital setting and ED [19, 49]. However, it must be noted that despite its clear and simple guidance, the WHO ladder does not consider newer medications and was developed to address cancer-related pain rather than being specific for emergency trauma pain [51, 52]. Indeed, a meeting of experts in 2007 urged the WHO to develop guidelines specific for trauma pain in emergency settings [52].

It was noted in some studies identified in this literature analysis that individual countries and institutions have developed their own protocols

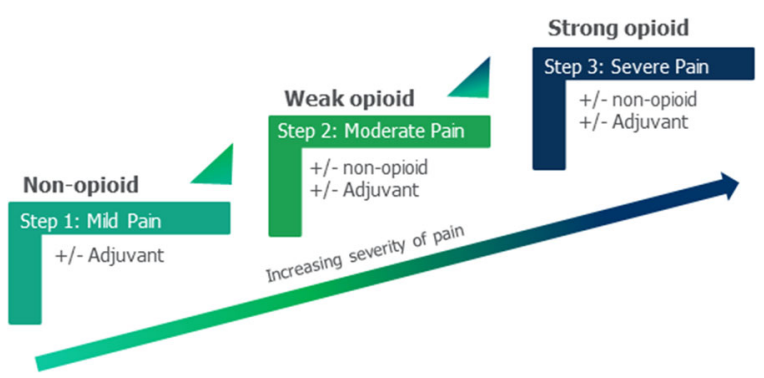

Fig. 3 Pathway for the treatment of pain (adapted from the WHO cancer pain ladder) [50] for pain management $[9,11,14,19]$. However, while details of institution-specific protocols are not widely published, these guidelines are likely to be inconsistent, which is supported by variations in the most common types of analgesia administered to treatment trauma pain across European countries [7, 19, 23, 30]. There are also numerous reports that adherence to pain management protocols in Europe is lacking (Fig. 2) [3, 11, 14, 30, 53]. Consequently, the development and implementation of effective pan-European and/or national guidelines is required to provide a clear process for the management of trauma pain and reduce discrepancies in treatment which may lead to differing levels of pain relief in patients $[14,18,53]$.

\section{Inadequate Assessment of Trauma Pain}

Evaluation of patient pain is vital for the implementation of effective pain management protocols $[5,8]$. A number of instruments have been developed to aid the evaluation of pain, such as the visual analogue scale (VAS) and numerical rating scale (NRS), which are commonly used in emergency settings [54]. Most of the studies which evaluated pain in this literature analysis utilized a NRS [3, 9-11, 20, 23, 41]. It should be noted that pain assessment scales are associated with limitations, measurements are not interchangeable and some are better suited to research rather than clinical practice settings [54]. However, when used in conjunction with clinical observations relating to pain intensity, these instruments provide HCPs with an objective measure of patient pain and in assist them to select the most appropriate analgesic.

Our literature search indicated that inadequate assessment of pain in emergency settings is common in Europe, which has, in part, been attributed to time constraints and ambiguity in protocols [3, 11, 31, 53]. If pain is not assessed frequently it cannot be comprehensively treated; consequently under evaluation of pain will result in undertreatment of some patients [4]. Guidance on the use of pain assessment tools is often specified to the local level, although may be based on national or international recommendations $[8,11,19]$. 


\section{Aversion to Opioid Analgesia}

The limitations associated with opioid analgesia discussed previously result in an aversion to opioid use by some patients and HCPs [14]. For example, in an Italian hospital emergency department, despite $77 \%$ of patients reporting severe pain which warranted opioid therapy per the center's pain management protocol, this was administered to only $3 \%$ of patients [20]. HCP aversion to administering opioid analgesia has been attributed to regulatory barriers in prescription, concern over patient drug-seeking behavior or addiction, increased demands due to patient monitoring, and fear of masking other symptoms of trauma [14, 30, 41, 53]. The phenomenon of 'opioid aversion' is also widely documented in studies conducted in other regions $[6,55]$. Opioids can provide potent analgesia when appropriately prescribed for moderate-to-severe pain, and consequently aversion to opioid use can result in the undertreatment of trauma pain. HCPs' reluctance to prescribe and administer opioids directly hinders pain management in both prehospital and ED settings. Across a range of settings, some patients also express concerns regarding opioid treatment and desire to be offered non-opioid pain relief [56].

When used appropriately, opioids present an effective treatment for severe trauma pain. Overcoming physician and patient aversion to opioid use may therefore reduce the burden of trauma pain by providing an effective treatment. This could potentially be achieved by the development of evidence-based national treatment guidelines which clearly document appropriate use of opioid analgesics for shortterm administration to address acute trauma pain, including patient selection. Readily available information on the risks of opioid-related side effects, overdose, diversion, and dependency associated with acute use could also be useful to assist HCPs to make informed decisions in emergency trauma settings, since much of the published literature on these issues pertains to chronic opioid therapy $[57,58]$.

\section{Delay to Administration of Analgesia}

Time to analgesia is critical in the treatment of trauma pain as delays result in undue patient suffering [5]. Reports that patients in pain upon arrival at ED experience more pain during examinations and procedures than other individuals, which may in part by attributed to hyperalgesia, also underscores the need for prompt administration of effective analgesia [4]. Data from real-world studies included in this literature analysis indicate that many patients with moderate-to-severe trauma pain do not receive analgesia within the 15 to 20-min timeframe suggested in the two local European guidelines identified $[8,19]$. For example, in a French observational study, $35 \%$ of patients waited for more than 60 min before examination, and average waiting times in excess of 40 min were reported in other audits conducted in hospitals in France and Portugal $[4,18,59]$. Similarly, in a prehospital setting, an average of 38 min was reported between paramedic arrival and administration of analgesia in a pan-European audit of patients with emergency trauma pain [60]. A critical area for improvement in time to analgesia in the ED is in triage time. Patients' first demonstration of pain in the ED presents an opportunity to provide appropriate analgesia, so a delay in this process presents a barrier to timely pain relief [59]. Triage systems used in EDs typically include an assessment of patients' pain to guide analgesia use and prioritize patients for treatment $[4,8,20,61]$. The Manchester Triage System has been utilized and adapted by many European countries (for example, Germany and Switzerland), where patients are assigned a triage category and corresponding target time to assessment [61]. Triage assessment of pain in European hospital EDs has been shown to result in rapid and effective analgesia, although some studies indicate failings in implementation of triage protocols as patients continue to receive suboptimal analgesia $[4,9,20,62]$.

Attitudes of some HCPs towards pain in emergency settings can also delay administration of analgesia, for example that pain is a minor priority in trauma care as it is not lifethreatening [53]. Studies conducted in the US indicate that overcrowding in the ED contributes to a prolonged time to analgesia as HCPs experience increased time pressures. Increased patient numbers in the ED can result 
in increased time to patient assessment, time to analgesic ordering, and time to administration of analgesia [63-65]. Time to analgesia also presents a barrier to effective pain relief in prehospital emergency settings. For example, short time at the scene and secondary missions were associated with untreated persistent pain in patients with moderate-to-severe pain attended by a helicopter emergency medical service [12]. The form of analgesia administered in ambulances can also influence the burden of trauma pain, as IV analgesia can increase on-scene time due to administration, resulting in longer mission times [42]. Furthermore, in some European countries, ambulance personnel are not permitted to administer opioid analgesics, although a recent study in Germany supported prehospital fentanyl and morphine administered by specially trained paramedics [66]. When pain cannot be managed effectively in pre-hospital settings, this also results in a greater burden for HCPs when patients arrive at the ED. The metabolism of analgesics can also influence the time taken to achieve pain relief, an important factor in emergency settings, and consequently sources of variability that may influence pharmacokinetics and pharmacodynamics should be considered (Fig. 2) [67].

\section{DISCUSSION}

Pain imposes a substantial burden on emergency care, as this is often the primary complaint of patients presenting to EDs [59]. We have identified a variety of limitations associated with many analgesics, including difficulties associated with IV administration necessary for some drugs and bulky equipment requirements for $\mathrm{N}_{2} \mathrm{O}$, particularly in pre-hospital settings $[34,43,44]$. Other treatment-associated limitations identified include aversion to opioid analgesics due to perceptions associated with this class of agents, safety concerns, and regulatory barriers [14, 41, 53]. Consequently, for the treatment of moderate-to-severe trauma pain in emergency settings, there remains an unmet need for analgesic agents to be widely used that have a fast onset of action, limited contraindications, and are easy to administer.
Barriers to the effective management of trauma pain in emergency settings outside of the analgesic products used were also identified. These included failure to use validated pain scales to aid triage assessment as well as low prioritization of trauma pain by some HCPs $[11,53]$. Furthermore, our literature search identified no pan-European clinical guidelines addressing management of trauma pain in emergency settings and only two local guidelines $[8,19]$. However, several publications referred to institution-specific protocols $[9,11,14]$. Consequently, development of national and regional European guidelines detailing analgesic use and the wider management of trauma pain in Europe would be key in reducing the burden of pain to HCPs $[52,55]$. Such guidelines should also include methods to accurately assess patient pain. Therefore, we recommend that relevant professional organizations across Europe who represent HCPs treating patients with trauma pain convene to develop clinical practice guidelines. Indeed, input from global experts should also be considered for best-practice recommendations. The findings from this European-focused literature analysis on trauma pain are supported by USfocused and global literature reviews, which call for timely assessment of pain at presentation and following administration of analgesia using age-appropriate, validated scales, and wider implementation of pain management protocols $[24,55,68]$.

Some barriers to the effective management of trauma pain in emergency settings could be addressed by use of easily portable, IN, nonopioid analgesia such as methoxyflurane $[34,35]$. Furthermore, by multimodal analgesia, i.e., multiple complementary analgesic agents used in combination, physicians can ensure that patients achieve adequate pain relief throughout their journey in emergency settings and possibly reduce the side effects associated with strong analgesics such as opioids [69]. The use of multimodal analgesia also allows the physician to tailor pain relief to an individual patient [69]. Patient-controlled analgesia may provide a solution to dosage and frequency limitations of current methods of pain relief $[70,71]$. 
The literature review was limited by only searching for articles in the English language, which may have discounted local language publications and guidelines. This review also focused solely on the treatment of adult patients with trauma pain and so does not discuss the separate challenges faced when treating elderly or pediatric patients (such as the likelihood of comorbidities and analgesic dosing considerations). Some publications identified from the literature review also included discussion of acute pain, not always as a result of trauma. These publications were captured as they provide a valuable insight into the barriers to effective management of acute pain in emergency settings. Furthermore, while this publication discusses the burden of trauma pain in Europe as a whole, due to the limited results of the literature search, information from all European countries could not be included.

\section{CONCLUSIONS}

In conclusion, based on evidence in published literature, the management of trauma pain in emergency settings across Europe could be improved by the development of novel analgesics and greater uptake of available agents, which overcome several of the practical and safety limitations associated with widely used products. Improved measures of assessing patient pain and the development and implementation of effective protocols for pain management will also be important steps in reducing the burden of trauma pain in emergency settings in Europe.

\section{ACKNOWLEDGEMENTS}

Funding. Article processing charges for this review paper were funded by Mundipharma International Ltd. All authors had full access to all of the data in this study and take complete responsibility for the integrity of the data and accuracy of the data analysis.
Editorial Assistance. The authors would like to acknowledge Hannah Collings and Ashley Enstone (Adelphi Values PROVE) for their contribution to developing the literature search strategy, conducting the literature review, and drafting the manuscript, funded by Mundipharma International Ltd. Editorial assistance in the preparation of this article was also provided by Siân Marshall of SIANTIFIX Ltd, Cambridgeshire, UK, funded by Mundipharma International Ltd.

Authorship. All named authors meet the International Committee of Medical Journal Editors (ICMJE) criteria for authorship for this article, take complete responsibility for the integrity and accuracy of this work as a whole, and have given their approval for this version to be published.

Disclosures. Patrick D. Dißmann received a consultancy fee and travel expenses from Mundipharma International Ltd. Maxime Maignan received a consultancy fee and travel expenses from Mundipharma International Ltd. Paul D. Cloves received a consultancy fee and travel expenses from Mundipharma International Ltd. Blanca Gutierrez Parres received a consultancy fee and travel expenses from Mundipharma International Ltd. Sara Dickerson is an employee of Mundipharma International Limited. Alice Eberhardt is an employee of Mundipharma GmbH.

Compliance with Ethics Guidelines. This article is based on previously conducted studies and does not contain any studies with human participants or animals performed by any of the authors.

Open Access. This article is distributed under the terms of the Creative Commons Attribution-NonCommercial 4.0 International License (http://creativecommons.org/licenses/ by-nc/4.0/), which permits any noncommercial use, distribution, and reproduction in any medium, provided you give appropriate credit to the original author(s) and the source, provide a link to the Creative Commons license, and indicate if changes were made. 


\section{REFERENCES}

1. EuroSafe. Injuries in the European Union: Summary of injury statistics for the years 2012-2014. Issue 6 . 2016. http://www.eurosafe.eu.com/uploads/inlinefiles/EuropeSafe_Master_Web_02112016\%20\%282 \%29.pdf. Accessed 1 June 2018

2. Berben SA, Meijs TH, van Dongen RT, et al. Pain prevalence and pain relief in trauma patients in the Accident \& Emergency department. Injury. 2008;39(5):578-85.

3. Berben SAA, Schoonhoven L, Meijs THJM, van Vugt $A B$, van Grunsven PM. Prevalence and relief of pain in trauma patients in emergency medical services. Clin J Pain. 2011;27(7):587-92.

4. Karwowski-Soulie F, Lessenot-Tcherny S, LamarcheVadel A, et al. Pain in an emergency department: an audit. Eur J Emerg Med. 2006;13(4):218-24.

5. Parker M, Rodgers A. Management of pain in prehospital settings. Emerg Nurse. 2015;23(3):16-21 (quiz 3).

6. Todd K, Ducharme J, Choiniere M, Crandall C, Fosnocht D, Homel P. Pain in the emergency department: results of the pain and emergency medicine initiative (PEMI) multicenter study. J Pain. 2007;8(6):460-6.

7. Boccard E, Adnet F, Gueugniaud PY, Filipovics A, Ricard-Hibon A. Pain management in adult patients in emergency care units in France in 2010. [French, English]. Annales Francaises de Medecine d'Urgence. 2011;1(5):312-9.

8. The College of Emergency Medicine. Management of pain in adults 2014. https://www.rcem.ac.uk/ docs/College\%20Guidelines/5w.\%20Management $\%$ 20of\%20Pain\%20in\%20Adults\%20(Revised\%20Dec ember\%202014).pdf. Accessed 1 June 2018

9. Van Woerden G, Van Den Brand CL, Den Hartog CF, Idenburg FJ, Grootendorst DC, Van Der Linden MC. Increased analgesia administration in emergency medicine after implementation of revised guidelines. Int J Emerg Med. 2016;9(1):4.

10. Mura P, Serra E, Marinangeli F, et al. Prospective study on prevalence, intensity, type, and therapy of acute pain in a second-level urban emergency department. J Pain Res. 2017;10:2781-8.

11. Dale J, Bjornsen L. Assessment of pain in a Norwegian emergency department. Scand J Trauma Resusc Emerg Med. 2015;23(1):86.

12. Oberholzer N, Kaserer A, Albrecht R, et al. Factors influencing quality of pain management in a physician-staffed helicopter emergency medical service. Anesth Analg. 2017;125(1):200-9.

13. Whittemore $\mathrm{R}$, Knafl $\mathrm{K}$. The integrative review: updated methodology. J Adv Nurs. 2005;52(5):546-53.

14. Stephan FP, Nickel CH, Martin JS, Grether D, Delport-Lehnen K, Bingisser R. Pain in the emergency department: adherence to an implemented treatment protocol. Swiss Med Wkly. 2010;140(23-24):341-7.

15. Vidouris A, Lin R, Bedford J, Amoako D. To block or not to block? The utilisation of regional anaesthesia on the trauma list in a London major trauma centre. Anaesthesia. 2016;71(Supplement 4):73.

16. Singh A, McGhee L, McNally D, Misra U. Audit of fascia iliaca blocks for hip fracture patients in Sunderland A\&E. Anaesthesia. 2017;17(Supplement 3):100.

17. Chawla S, Chawla M, Venkataraju A. Pain management in hip fracture: a service evaluation. Anaesthesia. 2017;72(Supplement 3):15.

18. Carreira CR, Oliveira E, Marques A, Valentim A. How pain is treated in an emergency department in a central hospital? A prospective study. Reg Anesth Pain Med. 2012;1:E213-4.

19. Tamches E, Buclin T, Hugli O, et al. Acute pain in adults admitted to the emergency room: development and implementation of abbreviated guidelines. Swiss Med Wkly. 2007;137:223-7.

20. Butti L, Bierti O, Lanfrit R, et al. Evaluation of the effectiveness and efficiency of the triage emergency department nursing protocol for the management of pain. J Pain Res. 2017;10:2479-88.

21. NHS. Non-steroidal anti-inflammatory drugs (NSAIDS) 2016 [Updated 22 March 2016]. http:// www.nhs.uk/conditions/Anti-inflammatories-nonsteroidal/Pages/Introduction.aspx\#examples. Accessed 1 June 2018

22. Ridderikhof ML, Lirk $\mathrm{P}$, Goddijn $\mathrm{H}$, et al. Acetaminophen or nonsteroidal anti-inflammatory drugs in acute musculoskeletal trauma: a multicenter, double-blind, randomized, clinical trial. Ann Emerg Med. 2018;71(3):357-68.

23. Ducasse JL, Siksik G, Durand-Bechu M, et al. Nitrous oxide for early analgesia in the emergency setting: a randomized, double-blind multicenter prehospital trial. Acad Emerg Med. 2013;20(2):178-84.

24. Thomas SH. Management of pain in the emergency department. ISRN Emerg Med. 2013;2013:19. 
25. Kotter T, da Costa BR, Fassler M, et al. Metamizoleassociated adverse events: a systematic review and meta-analysis. PLoS One. 2015;10(4):e0122918.

26. Jasiecka A, Maslanka T, Jaroszewski JJ. Pharmacological characteristics of metamizole. Pol J Vet Sci. 2014;17(1):207-14.

27. Andrade S, Bartels DB, Lange R, Sandford L, Gurwitz J. Safety of metamizole: a systematic review of the literature. J Clin Pharm Ther. 2016;41(5):459-77.

28. Keene DD, Rea WE, Aldington D. Acute pain management in trauma. Trauma. 2011;13(3):167-79.

29. Pergolizzi JV Jr, van de Laar M, Langford R, et al. Tramadol/paracetamol fixed-dose combination in the treatment of moderate-to-severe pain. J Pain Res. 2012;5:327-46.

30. Marinangeli F, Narducci C, Ursini ML, et al. Acute pain and availability of analgesia in the prehospital emergency setting in Italy: a problem to be solved. Pain Pract. 2009;9(4):282-8.

31. Hebsgaard S, Mannering A, Zwisler ST. Assessment of acute pain in trauma: a retrospective prehospital evaluation. J Opioid Manag. 2016;12(5):347-53.

32. Jonkman K, Dahan A, van de Donk T, Aarts L, Niesters M, van Velzen M. Ketamine for pain. F1000Res. 2017. https://doi.org/10.12688/f1000rese arch.11372.1

33. Dayan AD. Analgesic use of inhaled methoxyflurane: evaluation of its potential nephrotoxicity. Hum Exp Toxicol. 2016;35(1):91-100.

34. Porter KM, Siddiqui MK, Sharma I, Dickerson S, Eberhardt A. Management of trauma pain in the emergency setting: low-dose methoxyflurane or nitrous oxide? A systematic review and indirect treatment comparison. J Pain Res. 2018;11:11-21.

35. Blair HA, Frampton JE. Methoxyflurane inhalation vapour in trauma pain: a profile of its use in the EU. Drugs Ther Perspect. 2017;33:403-8.

36. Coffey F, Wright J, Hartshorn S, et al. STOP!: a randomised, double-blind, placebo-controlled study of the efficacy and safety of methoxyflurane for the treatment of acute pain. Emerg Med J. 2014;31(8):613-8.

37. Coffey F, Dissmann P, Mirza K, Lomax M. Methoxyflurane analgesia in adult patients in the emergency department: a subgroup analysis of a randomized, double-blind, placebo-controlled study (STOP!). Adv Ther. 2016;33(11):2012-31.

38. Dissmann P, Coffey F, Mirza K, Lomax M. Global medication performance and safety of methoxyflurane analgesia in adult patients with contusions and lacerations treated in the emergency department. Crit Care. 2017;21(Supplement $1): 38$.

39. Coffey F, Dissmann P, Mirza K, Lomax M. The duration of methoxyflurane analgesia in adult patients in the emergency department: a sub-analysis of STOP!, a randomised, double-blind, placebo-controlled study. Crit Care. 2017;21(Supplement 1):38.

40. Dahl JB, Nielsen RV, Wetterslev J, et al. Post-operative analgesic effects of paracetamol, NSAIDs, glucocorticoids, gabapentinoids and their combinations: a topical review. Acta Anaesthesiol Scand. 2014;58(10):1165-81.

41. Friesgaard KD, Nikolajsen L, Giebner M, et al. Efficacy and safety of intravenous fentanyl administered by ambulance personnel. Acta Anaesthesiol Scand. 2016;60:537-43.

42. Buntine $\mathrm{P}$, Thom O, Babl F, Bailey M, Bernard S. Prehospital analgesia in adults using inhaled methoxyflurane. Emerg Med Australas. 2007;19(6):509-14.

43. Fields JM, Piela NE, Ku BS. Association between multiple IV attempts and perceived pain levels in the emergency department. J Vasc Access. 2014;15(6):514-8.

44. Witting MD. IV access difficulty: incidence and delays in an urban emergency department. J Emerg Med. 2012;42:483-7.

45. Hansen MS, Dahl JB. Limited evidence for intranasal fentanyl in the emergency department and the prehospital setting: a systematic review. Dan Med J. 2013;60(1):A4563.

46. Oyler DR, Parli SE, Bernard AC, Chang PK, Procter LD, Harned ME. Nonopioid management of acute pain associated with trauma: focus on pharmacologic options. J Trauma Acute Care Surg. 2015;79(3):475-83.

47. NHS. Paracetamol for adults 2014 [Updated 18 May 2016]. https://beta.nhs.uk/medicines/paracetamolfor-adults. Accessed 1 June 2018

48. Roberts E, Delgado Nunes V, Buckner S, et al. Paracetamol: not as safe as we thought? A systematic literature review of observational studies. Ann Rheum Dis. 2016;75(3):552-9.

49. Gausche-Hill M, Brown KM, Oliver ZJ, et al. An evidence-based guideline for prehospital analgesia in trauma. Prehosp Emerg Care. 2014;18(Suppl 1):25-34.

50. World Health Organization. WHO's cancer pain ladder for adults 1986. http://www.who.int/cancer/ palliative/painladder/en/. Accessed 1 June 2018 
51. Vargas-Schaffer G. Is the WHO analgesic ladder still valid? Twenty-four years of experience. Can Fam Physician. 2010;56(6):514-7 (e202-5).

52. World Health Organization. WHO normative guidelines on pain management 2007. http://www. who.int/medicines/areas/quality_safety/delphi_stu dy_pain_guidelines.pdf. Accessed 1 June 2018

53. Berben SAA, Meijs THJM, Van Grunsven PM, Schoonhoven L, Van Achterberg T. Facilitators and barriers in pain management for trauma patients in the chain of emergency care. Injury. 2012;43(9): 1397-402.

54. Kumar P, Tripathi L. Challenges in pain assessments: pain intensity scales. Indian J Pain. 2014;28:61-70.

55. Motov SM, Khan ANGA. Problems and barriers of pain management in the emergency department: are we ever going to get better? J Pain Res. 2009;2:5-11.

56. Sinatra R. Causes and consequences of inadequate management of acute pain. Pain Med. 2010;11(12):1859-71.

57. Volkow ND, McLellan AT. Opioid abuse in chronic pain: misconceptions and mitigation strategies. N Engl J Med. 2016;374(13):1253-63.

58. Atluri S, Akbik H, Sudarshan G. Prevention of opioid abuse in chronic non-cancer pain: an algorithmic, evidence based approach. Pain Physician. 2012;15(3 Suppl):ES177-89.

59. Kone V, Lecomte F, Randriamanana D, Pourriat JL, Claessens YE, Vidal-Trecan G. Impact of a pilot team on patients' pain reduction and satisfaction in an emergency department: a before-and-after observational study. Rev Epidemiol Sante Publique. 2016;64:59-66.

60. Xia A, Nokela M, Liebmeier M, Szende A, Colman S. Pre-hospital pain relief treatment in patients with musculoskeletal injuries experiencing moderate-tosevere pain in medical emergencies. Value in Health. 2017;20:A508.

61. Steiner D, Renetseder F, Kutz A, et al. Performance of the Manchester Triage System in adult medical emergency patients: a prospective cohort study. J Emerg Med. 2016;50(4):678-89.
62. Dilworth S, Sweeney M. Management of acute pain in adults attending an emergency department: a closed loop audit. Ir J Med Sci. 2017;186(Supplement 6):S225-6.

63. Hwang U, Richardson L, Livote E, Harris B, Spencer $\mathrm{N}$, Sean Morrison R. Emergency department crowding and decreased quality of pain care. Acad Emerg Med. 2008;15:1248-55.

64. Pines JM, Hollander JE. Emergency department crowding is associated with poor care for patients with severe pain. Ann Emerg Med. 2008;51(1):1-5.

65. Mills AM, Shofer FS, Chen EH, Hollander JE, Pines JM. The association between emergency department crowding and analgesia administration in acute abdominal pain patients. Acad Emerg Med. 2009;16(7):603-8.

66. Scharonow M, Alberding T, Oltmanns W, Weilbach C. Project for the introduction of prehospital analgesia with fentanyl and morphine administered by specially trained paramedics in a rural service area in Germany. J Pain Res. 2017;10:2595-9.

67. Martini C, Olofsen E, Yassen A, Aarts L, Dahan A. Pharmacokinetic-pharmacodynamic modeling in acute and chronic pain: an overview of the recent literature. Expert Rev Clin Pharmacol. 2011;4(6): 719-28.

68. Ahmadi A, Bazargan-Hejazi S, Heidari Zadie Z, et al. Pain management in trauma: a review study. J Inj Violence Res. 2016;8(2):89-98.

69. Elvir-Lazo OL, White PF. The role of multimodal analgesia in pain management after ambulatory surgery. Curr Opin Anaesthesiol. 2010;23(6): 697-703.

70. Rahman NH, DeSilva T. A randomized controlled trial of patient-controlled analgesia compared with boluses of analgesia for the control of acute traumatic pain in the emergency department. J Emerg Med. 2012;43(6):951-7.

71. Smith JE, Rockett M, Creanor S, et al. PAin SoluTions In the Emergency Setting (PASTIES): patient controlled analgesia versus routine care in emergency department patients with pain from traumatic injuries: randomised trial. BMJ. 2015;350:h2988. 